\title{
EDA, EDAR, EDARADD and WNT10A allelic variants in patients with ectodermal derivative impairment in the Spanish population
}

María Carmen Martínez-Romero 1,2, María Juliana Ballesta-Martínez,4, Vanesa López-González 3,4, María José Sánchez-Soler ${ }^{3,4}$, Ana Teresa Serrano-Antón ${ }^{3}$, María Barreda-Sánchez ${ }^{4}$, Lidya Rodriguez-Peña ${ }^{3}$, María Teresa Martínez-Menchon ${ }^{5}$, José Frías-Iniesta ${ }^{5}$, Paloma Sánchez-Pedreño ${ }^{5}$, Pablo Carbonell-Meseguer ${ }^{1}$, Guillermo Glover-López ${ }^{1}$, Encarna Guillén-Navarro ${ }^{6,7^{*}}$ [D and GIEDE (Spanish multidisciplinary research group for ectodermal dysplasia)

\begin{abstract}
Background: Ectodermal dysplasias (ED) are a group of genetic conditions affecting the development and/or homeostasis of two or more ectodermal derivatives. An attenuated phenotype is considered a non-syndromic trait when the patient is affected by only one impaired ectodermal structure, such as in non-syndromic tooth agenesis (NSTA) disorder. Hypohidrotic ectodermal dysplasia (HED) is the most highly represented ED. X-linked hypohidrotic ectodermal dysplasia (XLHED) is the most common subtype, with an incidence of 1/50,000-100,000 males, and is associated with the EDA gene (Xq12-q13.1); the dominant and recessive subtypes involve the EDAR (2q13) and EDARADD (1q42.3) genes, respectively. The WNT10A gene (2q35) is associated more frequently with NSTA. Our goal was to determine the mutational spectrum in a cohort of 72 Spanish patients affected by one or more ectodermal derivative impairments referred to as HED (63/72) or NSTA (9/72) to establish the prevalence of the allelic variants of the four most frequently associated genes. Sanger sequencing of the EDA, EDAR, EDARADD and WNT10A genes and multiplex ligation-dependent probe amplification (MLPA) were performed.
\end{abstract}

Results: A total of 61 children and 11 adults, comprising 50 males and 22 females, were included. The average ages were 5.4 and 40.2 years for children and adults, respectively. A molecular basis was identified in 51/72 patients, including 47/63 HED patients, for whom EDA was the most frequently involved gene, and 4/9 NSTA patients, most of whom had variants of WNT10A. Among all the patients, 37/51 had variants of EDA, 8/51 had variants of the WNT10A gene, 4/51 had variants of EDAR and 5/51 had variants of EDARADD. In 42/51 of cases, the variants were inherited according to an X-linked pattern (27/42), with the remaining showing an autosomal dominant (10/42) or autosomal recessive (5/42) pattern. Among the NSTA patients, 3/9 carried pathogenic variants of WNT10A and 1/9 carried EDA variants. A total of 60 variants were detected in 51 patients, 46 of which were different, and out of these 46 variants, 12 were novel.

(Continued on next page)

\footnotetext{
* Correspondence: encarna.guillen@carm.es; eguillen@um.es

${ }^{6}$ Departamento de Cirugía, Pediatría, Obstetricia y Ginecología. Facultad de Medicina, Universidad de Murcia, Murcia, Spain

${ }^{7}$ Sección Genética Médica (Hospital Materno-Infantil. Planta 0), Hospital

Clínico Universitario Virgen de la Arrixaca, Ctra. Madrid-Cartagena s/n, El

Palmar, CP 30120 Murcia, Spain

Full list of author information is available at the end of the article
}

(C) The Author(s). 2019 Open Access This article is distributed under the terms of the Creative Commons Attribution 4.0 International License (http://creativecommons.org/licenses/by/4.0/), which permits unrestricted use, distribution, and reproduction in any medium, provided you give appropriate credit to the original author(s) and the source, provide a link to the Creative Commons license, and indicate if changes were made. The Creative Commons Public Domain Dedication waiver (http://creativecommons.org/publicdomain/zero/1.0/) applies to the data made available in this article, unless otherwise stated. 
(Continued from previous page)

Conclusions: This is the only molecular study conducted to date in the Spanish population affected by ED. The EDA, EDAR, EDARADD and WNT10A genes constitute the molecular basis in $70.8 \%$ of patients with a $74.6 \%$ yield in HED and $44.4 \%$ in NSTA. Twelve novel variants were identified. The WNT10A gene has been confirmed as the second molecular candidate that has been identified and accounts for one-half of non-EDA patients and one-third of NSTA patients. Further studies using next generation sequencing (NGS) will help to identify other contributory genes in the remaining uncharacterized Spanish patients.

Keywords: Ectodermal derivative impairment, hypohidrotic ectodermal dysplasia, Non-syndromic tooth agenesis, Hypodontia, EDA, EDAR, EDARADD, WNT10A

\section{Background}

Ectodermal dysplasias (ED) are a group of genetic conditions affecting the development and/or homeostasis of two or more ectodermal derivatives. An attenuated phenotype has been considered to be a non-syndromic trait when the patient is affected by only one impaired ectoderm-derived structure [1]. ED is a large and heterogeneous group of congenital disorders affecting the normal development of ectoderm-derived structures such as hair, nails, teeth and eccrine sweat glands [2-6]. Hypohidrotic ectodermal dysplasia (HED) (OMIM: \# 305100; ORPHA: 238468) is the most common type of ED. HED is due to pathogenic variants in several genes that encode components of the tumour necrosis factor $\alpha$ (TNF $\alpha$ )-related signalling pathway [7]. Pathogenic variants of these genes interfere with the interaction between surface-localized epithelial cells and the underlying mesenchyme, which occurs during embryonic development [8].

It has been demonstrated that HED is caused by pathogenic variants in several genes, including EDA (OMIM 300451), which is located on chromosome Xq12-q13.3 and encodes the ligand ectodysplasinA-A1 (EDA-A1), EDAR (OMIM 604095), which is located on chromosome 2q11-13 and encodes the ectodysplasinAA1 receptor, and EDARADD (OMIM 606603), which is located on chromosome 1q42-q43 and regulates the structure of EDAR-associated death domain protein. With the exception of the EDA gene, which is located on the $\mathrm{X}$ chromosome, all other genes encoding components of the TNF $\alpha$-related signalling pathway involved in the differentiation of skin appendages are located on the autosomes.

Pathogenic variants of the WNT10A gene (chromosome 2q35, OMIM 606268) are involved in the impairment of one or more ectodermal derivatives that cause HED, odonto-onycho-dermal dysplasia (OODD) (OMIM: \# 257980; ORPHA: 2721), Schöpf-Schulz-Passarge syndrome (SSPS) (OMIM: \# 224750; ORPHA: 50944) [9] and syndromic or non-syndromic NSTA (ORPHA: 99798) [10]. EDA pathogenic variants that underlie non-syndromic or syndromic oligodontia [11] have been described, likely because they alter a single signal transduction pathway. It has been postulated that X-linked hypohidrotic ectodermal dysplasia (XLHED) and EDA-related NSTA are the same disease with different degrees of expressivity [12].

In this study, we evaluated, for the first time, the mutational spectrum in the Spanish population with impairment of one or more ectodermal derivatives. We completely sequenced the EDA,EDAR, EDARADD and WNT10A genes in a large cohort of 72 unrelated patients.

\section{Materials and methods Subjects}

A cohort of 72 Spanish subjects from a multicenter cross-sectional study with clinical signs of ectodermal derivative impairment was recruited; 63 (87.5\%) cases were referred to as HED, and 9 (12.5\%) were referred to as non-syndromic tooth agenesis (NSTA). Among these, 50 involved males and 22 involved females with ages ranging from 6 months to 69 years, most of whom were children (<18 years) (84.7\%). Clinical data and family history were collected with a specific questionnaire, and blood samples for genetic analysis were collected after written informed consent was obtained from patients or, in the case of minors, from their parents. The study was approved by the Virgen de la Arrixaca University Hospital Ethical Committee and adhered to the Helsinki Declaration.

\section{Sequencing analysis}

Genomic DNA was extracted from peripheral blood lymphocytes using the commercial Maxwell ${ }^{\bullet} 16$ blood DNA purification kit (Promega Corporation, Madison, WI, USA) with the automatized system Maxwell $^{\circledR} 16$ (Promega Corporation, Madison, WI, USA). Primers covering the exons and a minimum of $20 \mathrm{bp}$ in the flanking intronic sequences of the EDA,EDAR, EDARADD and WNT10A genes were designed with Prime3Plus [13]. PCR was performed, and the products were sequenced with an ABI3100 genetic analyser (Applied Biosystems, Foster City, CA, USA). The electropherograms were analysed 
with Sequence Scape Software v3.0 (Applied Biosystems, Foster City, CA, USA). A mutation nomenclature was used in which +1 corresponded to the A of the ATG translation initiation codon of the reference sequence NM_001399.4 $(E D A)$, NM_022336.3 (EDAR), NM_145861.2 (EDARADD) or NM_025216.2 (WNT10A). All detected variants were assessed using the Mutation Taster [14], SIFT [15], PolyPhen2 [16] and PROVEAN [17] in silico human genome variant prediction tools. The interpretation of the sequence variants was performed according to the American College of Medical Genetics and Genomics (ACMG) guidelines [18]. This study did not include causative mutations in remote intronic or regulatory regions of genes.

\section{Assessment of copy number variation by MLPA}

Multiple ligation-dependent probe amplification (MLPA) was performed with the SALSA MLPA P183-C1 EDAEDAR-EDARAD probe mix commercial kit and the SALSA MLPA EK1 Cy5 reagent kit (MRC-Holland, Amsterdam, Netherlands) according to the manufacturer's instructions. The fragment sizes were determined by capillary electrophoresis using the above-mentioned nucleic acid analyser. The relative exon copy numbers were calculated after normalization of the peak height value of the patients against the mean of the peak height value of the control group (at least two individuals of the same sex were known to have normal gene dosage) using the Coffalyser. Net software by MRC-Holland.

\section{Pattern of $\mathrm{X}$ inactivation}

The $\mathrm{X}$ inactivation patterns were analysed using an assay of the polymorphic CAG repeat in exon 1 of the androgen receptor gene (AR). The digestion of peripheral blood DNA with the methylation-sensitive restriction enzyme HpaII resulted in cleavage only in restriction sites of active $\mathrm{X}$-chromosomes, while the inactive $\mathrm{X}$ chromosomes remained intact. The direction of skewing was determined in female carriers when the parental mutation origin was confirmed by segregation analysis of the polymorphic repeat in AR. An X-chromosome inactivation pattern that was skewed $80: 20$ or less was classified as random, while a pattern skewed between 80 : 20 and 90:10 was classified as moderately skewed, and a pattern skewed more than 90:10 was considered highly skewed [19].

\section{Results}

The average age at genetic diagnosis was 5.4 years in children (42/51) and 40.2 years in adult patients (9/51). Pathogenic variants were identified in coding sequences or flanking intronic regions in $70.8 \%(51 / 72)$ of patients. Among the patients, $72.5 \%(37 / 51)$ had variants of $E D A$, $15.7 \%(8 / 51)$ had variants of the WNT10A gene, 7.8\% (4/ 51) had variants of EDAR and 9.8\% (5/51) had variants of $E D A R A D D$. In most cases, the variants were inherited $(82.4 \% ; 42 / 51)$, and the variants were inherited according to an X-linked (64.3\%; 27/42), autosomal dominant $(23.8 \% ; 10 / 42)$ or autosomal recessive pattern $(11.9 \%$; 5 / 42). Regarding the phenotype, pathogenic variants were identified in $74.6 \%(47 / 63)$ of HED patients and $44.4 \%$ (4/9) of NSTA patients. EDA was the most frequently involved gene in HED patients $(76.6 \% ; 36 / 47)$. In NSTA patients, WNT10A variants were present in 3 cases, and an $E D A$ variant was present in one case. A total of 46 different pathogenic variants (76.6\%) were identified among 60 variants in 51 patients. The allelic heterogeneity was $86.4 \%(32 / 37)$ for $E D A, 100 \%(5 / 5)$ for $E D A R$, $20 \%(1 / 5)$ for EDARADD and 66.6\% (8/13) for WNT10A. The observed variant types were complete EDA gene deletion [1], EDA exon 1 deletion [1], missense variants [20], nonsense variants [7] small indels [4], frameshift variants [6] and intronic variants [2]. Most pathogenic variants were located in conserved and functionally relevant domains in known hotspots. Twelve out of 46 variants $(26.0 \%)$ were novel and had not been previously reported among the 471 variant entries for these four genes in the Human Gene Mutation Database (HGMD ${ }^{\circledR}$ ) [21] (last consultation on August, 15, 2019).

$E D A$ was the most frequently involved gene (72.5\%), as previously mentioned, with pathogenic variants in 30 males and 7 females. Of these, 32 variants were different, and 9 were novel and more frequently located in exons $1,2,4,7$ and 8 . Seventy-five percent of variants were inherited (27/36) and most were found in only one family, except for the complete exon 1 deletion (two cases), c.572_598del18/p.Pro191_Pro196del (three cases), c.467G > A/p.Arg156His in the furin domain (two cases) and c.1045G > A/p.Ala349Thr (two cases). Regarding the predicted functional impact of the variants, three of them resulted in the complete disruption of the protein, and the rest were distributed within coding regions in the transmembrane domain [2], furin cleavage site [4], collagen-like domain [10] and tumour necrosis factor homology domain [15] (Table 1).

Male patients with $E D A$ pathogenic variants presented the classical XLHED phenotype. EDA female carriers also showed the HED phenotype; 6 out of 7 had predicted variants with severe functional consequences, including the disruption of the protein by frameshift mutations [3], partial or total EDA gene deletion [2] or a novel missense mutation affecting an amino acid (c.871G > C/ p.Gly291Arg) described as crucial for proper protein folding [25]. X-chromosome inactivation analysis was informative in 6 out of 7 female carriers and revealed a random pattern favouring either chromosome $(5 / 6)$ or moderately skewed X-chromosome inactivation (1/6). The HED phenotype in EDA female carriers was not correlated with the X-chromosome inactivation pattern (Table 2). 
Table 1 Allelic variants identified in the EDA gene

\begin{tabular}{|c|c|c|c|c|c|c|c|c|c|}
\hline Family & Gender & NM & $\begin{array}{l}\text { Exon/ } \\
\text { Intron }\end{array}$ & Protein & Affected domain & Variant & Origin & $\begin{array}{l}\text { Clinical } \\
\text { Diagnosis }\end{array}$ & $\operatorname{HGMD}(*)$ \\
\hline 10 & $\mathrm{~F}$ & Gene deletion & Complete & $(-)$ & Gene loss & Deletion & De novo & HED & {$[22]$} \\
\hline 13 & M & C. $58 \mathrm{C}>\mathrm{T}$ & Exon 1 & p.Arg20Ter & $\begin{array}{l}\text { Transmembrane } \\
\text { domain }\end{array}$ & Nonsense & Inherited & HED & [23] \\
\hline 26 & M & c. $164 \mathrm{~T}>\mathrm{C}$ & Exon 1 & p.Leu55Pro & $\begin{array}{l}\text { Transmembrane } \\
\text { domain }\end{array}$ & Missense & Inherited & HED & Novel \\
\hline 20 & M & $\begin{array}{l}\text { Exon } 1 \\
\text { deletion }\end{array}$ & Exon 1 & $(-)$ & Complete protein loss & Deletion & Inherited & HED & {$[24]$} \\
\hline 33 & $\mathrm{~F}$ & $\begin{array}{l}\text { Exon } 1 \\
\text { deletion }\end{array}$ & Exon 1 & $(-)$ & Complete protein loss & Deletion & De novo & HED & [24] \\
\hline 35 & M & $c .457 C>T$ & Exon 2 & p.Arg153Cys & Furin subdomain & Missense & De novo & HED & {$[25]$} \\
\hline 34 & M & c. $463 C>T$ & Exon 2 & p.Arg155Cys & Furin subdomain & Missense & De novo & HED & {$[26]$} \\
\hline 45 & M & c. $467 \mathrm{G}>\mathrm{A}$ & Exon 2 & p.Arg156His & Furin cleavage site & Missense & Inherited & HED & {$[26]$} \\
\hline 47 & M & $c .467 \mathrm{G}>\mathrm{A}$ & Exon 2 & p.Arg156His & Furin cleavage site & Missense & Inherited & HED & {$[26]$} \\
\hline 31 & $\mathrm{~F}$ & c.516delC & Exon 3 & p.Asn172Lysfs*108 & $(-)$ & Frame Shift & De novo & HED & Novel \\
\hline 17 & M & $\begin{array}{l}\text { c.546 } \\
\text { 581del36 }\end{array}$ & Exon 4 & $\begin{array}{l}\text { p.Asn185- } \\
\text { Pro196del }\end{array}$ & Collagen-like domain & $\begin{array}{l}\text { In-Frame } \\
\text { Deletion }\end{array}$ & Inherited & HED & {$[26]$} \\
\hline 37 & M & $\begin{array}{l}\text { c.553- } \\
\text { 588del36 }\end{array}$ & Exon 4 & $\begin{array}{l}\text { p.Asn185- } \\
\text { Pro196del }\end{array}$ & Collagen-like domain & $\begin{array}{l}\text { In-Frame } \\
\text { Deletion }\end{array}$ & Inherited & HED & {$[27]$} \\
\hline 21 & M & $\begin{array}{l}\text { c.572- } \\
\text { 589del18 }\end{array}$ & Exon 4 & $\begin{array}{l}\text { p.Pro191_- } \\
\text { Pro196del }\end{array}$ & Collagen-like domain & $\begin{array}{l}\text { In-Frame } \\
\text { Deletion }\end{array}$ & Inherited & HED & {$[28]$} \\
\hline 23 & M & $\begin{array}{l}\text { c.572- } \\
\text { 589del18 }\end{array}$ & Exon 4 & $\begin{array}{l}\text { p.Pro191_- } \\
\text { Pro196del }\end{array}$ & Collagen-like domain & $\begin{array}{l}\text { In-Frame } \\
\text { Deletion }\end{array}$ & Inherited & HED & {$[28]$} \\
\hline 48 & M & $\begin{array}{l}\text { c.572- } \\
\text { 589del18 }\end{array}$ & Exon 4 & $\begin{array}{l}\text { p.Pro191_- } \\
\text { Pro196del }\end{array}$ & Collagen-like domain & $\begin{array}{l}\text { In-Frame } \\
\text { Deletion }\end{array}$ & Inherited & HED & {$[28]$} \\
\hline 50 & M & c. $599 \mathrm{C}>\mathrm{G}$ & Exon 4 & p.Pro200Arg & Collagen-like domain & Missense & Inherited & HED & Novel \\
\hline 36 & $\mathrm{~F}$ & c.612delG & Exon 4 & $(-)$ & Collagen-like domain & Frame Shift & De novo & HED & Novel \\
\hline 02 & M & $\begin{array}{l}\text { c.612 } \\
\text { 629del18 }\end{array}$ & Exon 4 & p.lle205_Gly210del & Collagen-like domain & $\begin{array}{l}\text { In-Frame } \\
\text { Deletion }\end{array}$ & Inherited & HED & [29] \\
\hline 06 & M & c.653G > T & Exon 4 & p.Gly218Val & Collagen-like domain & Missense & Inherited & HED & {$[30]$} \\
\hline 01 & M & $\begin{array}{l}\text { c.663- } \\
\text { 697del35 }\end{array}$ & Exon 4 & p.Pro222Thrfs* & Collagen-like domain & Frame Shift & Inherited & HED & {$[26]$} \\
\hline 29 & M & $\begin{array}{l}\text { c. } 728 \text { - } \\
\text { 735del8 }\end{array}$ & Exon 5 & p.Thr243Lysfs*20 & $(-)$ & Frame Shift & De novo & HED & [24] \\
\hline 11 & $\mathrm{~F}$ & c.729_735del7 & Exon 5 & p.Arg244Thrfs*34 & $(-)$ & Frame Shift & Inherited & HED & Novel \\
\hline 14 & M & c.760C $>\mathrm{T}$ & Exon 6 & p.GIn $254^{*}$ & $\begin{array}{l}\text { TNF homology } \\
\text { domain }\end{array}$ & Nonsense & De novo & HED & Novel \\
\hline 22 & M & c. $793+1 G>C$ & Intron 6 & $(-)$ & $\begin{array}{l}\text { TNF homology } \\
\text { domain }\end{array}$ & Splicing & Inherited & HED & [25] \\
\hline 51 & M & $c .866 \mathrm{G}>\mathrm{A}$ & Exon7 & p.Gly289His & $\begin{array}{l}\text { TNF homology } \\
\text { domain }\end{array}$ & Missense & ND & NSTA & {$[31]$} \\
\hline 15 & $\mathrm{~F}$ & $c .871 G>C$ & Exon 7 & p.Gly291Arg & $\begin{array}{l}\text { TNF homology } \\
\text { domain }\end{array}$ & Missense & Inherited & HED & $\begin{array}{l}\text { Novel } \\
{[28](\#)}\end{array}$ \\
\hline 16 & $\mathrm{~F}$ & $c .880 G>T$ & Exon 7 & p.Glu294Ter & $\begin{array}{l}\text { TNF homology } \\
\text { domain }\end{array}$ & Nonsense & De novo & HED & Novel \\
\hline 07 & M & $c .892 \mathrm{G}>\mathrm{T}$ & Exon 7 & p.Asp298Tyr & $\begin{array}{l}\text { TNF homology } \\
\text { domain }\end{array}$ & Missense & Inherited & HED & {$[32]$} \\
\hline 08 & M & c. $895 \mathrm{G}>\mathrm{A}$ & Exon 7 & p.Gly299Ser & $\begin{array}{l}\text { TNF homology } \\
\text { domain }\end{array}$ & Missense & Inherited & HED & [26] \\
\hline 09 & M & c. $895 \mathrm{G}>\mathrm{C}$ & Exon 7 & p.Gly299Arg & $\begin{array}{l}\text { TNF homology } \\
\text { domain }\end{array}$ & Missense & Inherited & HED & [33] \\
\hline 03 & M & c. $995 \mathrm{G}>\mathrm{A}$ & Exon 8 & p.Cys332Tyr & TNF homology & Missense & Inherited & HED & [24] \\
\hline
\end{tabular}


Table 1 Allelic variants identified in the EDA gene (Continued)

\begin{tabular}{|c|c|c|c|c|c|c|c|c|c|}
\hline Family & Gender & NM & $\begin{array}{l}\text { Exon/ } \\
\text { Intron }\end{array}$ & Protein & Affected domain & Variant & Origin & $\begin{array}{l}\text { Clinical } \\
\text { Diagnosis }\end{array}$ & $\operatorname{HGMD}\left(^{*}\right)$ \\
\hline & & & & & domain & & & & \\
\hline 30 & M & c. $1027 \mathrm{~T}>\mathrm{G}$ & Exon 8 & p.Tyr343Asp & $\begin{array}{l}\text { TNF homology } \\
\text { domain }\end{array}$ & Missense & Inherited & HED & Novel \\
\hline 19 & M & c. $1037 \mathrm{G}>\mathrm{A}$ & Exon 8 & p.Cys346Tyr & $\begin{array}{l}\text { TNF homology } \\
\text { domain }\end{array}$ & Missense & Inherited & HED & [7] \\
\hline 24 & M & c. $1045 G>A$ & Exon 8 & p.Ala349Thr & $\begin{array}{l}\text { TNF homology } \\
\text { domain }\end{array}$ & Missense & Inherited & HED & [26] \\
\hline 04 & M & c. $1045 G>A$ & Exon 8 & p.Ala349Thr & $\begin{array}{l}\text { TNF homology } \\
\text { domain }\end{array}$ & Missense & Inherited & HED & [26] \\
\hline 32 & M & c. $1049 G>A$ & Exon 8 & p.Gly350Asp & $\begin{array}{l}\text { TNF homology } \\
\text { domain }\end{array}$ & Missense & Inherited & HED & [34] \\
\hline 25 & M & c. 1069 C > T & Exon 8 & p.Arg357Trp & $\begin{array}{l}\text { TNF homology } \\
\text { domain }\end{array}$ & Missense & Inherited & HED & [29] \\
\hline
\end{tabular}

$F$ female, $M$ male, TNF tumour necrosis factor, NM nucleotide mutation, HED hypohidrotic ectodermal dysplasia, $\left({ }^{*}\right)$ Reference in HGMD database, (\#) Novel variant in a nucleotide resulting in an equivalent amino acid, NSTA non-syndromic tooth agenesis, ND no data

$E D A R$ variants were detected in 4 HED patients. One of these was a compound heterozygous mutation comprising a splicing variant upstream of the first intron (c.52-2A > G) and a missense variant in the TNFR (tumour necrosis factor receptor) domain (c.212G > A/ p.Cys71Tyr) with an autosomal recessive inheritance pattern (published data) [3]; the other three variants, which were located in the death domain (DD), showed an autosomal dominant inheritance pattern (Table 3). All the $E D A R$ pathogenic variants had been reported previously in the HGMD. HED patients with EDAR pathogenic variants were clinically indistinguishable from those patients with $E D A$ variants. The Asian variant p.Val370Arg, which has been associated with an attenuated phenotype [35], was not observed in our cohort.

In $E D A R A D D$, the c.308C > T/ p.Ser103Phe variant, which has been described as likely to be benign in ClinVar and of unknown significance in the HGMD, was identified in 5 patients and was inherited in all cases (Table 4). Three of the patients carried additional variants; two were located in the EDA gene (c.572_589 del18 (family 21)) and were associated with the classical HED phenotype, and the other (c.866G > A (family 51) was identified in a patient with NSTA. In one female (family 28), an additional c. $682 \mathrm{~T}>\mathrm{C}$ variant in the WNT10A gene was linked to NSTA. In two HED patients, p.Ser103Phe in EDARADD was the only identified variant.

Table 2 X-chromosome inactivation pattern in EDA carrier females

\begin{tabular}{|c|c|c|c|c|c|c|c|c|}
\hline \multirow[t]{2}{*}{ Family } & \multirow[t]{2}{*}{ Case } & \multicolumn{3}{|c|}{$\mathrm{N}^{\circ}$ (CAG) repeat in AR (bp) } & \multicolumn{2}{|c|}{$\%$ Chromosome $X$ inactivation } & & \multirow{2}{*}{$\begin{array}{l}\text { Clinical } \\
\text { Diagnosis }\end{array}$} \\
\hline & & Variant & A1 & $\mathrm{A} 2$ & A1 & $\mathrm{A} 2$ & & \\
\hline 10 & Proband & EDA gene deletion & 270 & 270 & $\mathrm{NI}$ & $\mathrm{NI}$ & - & HED \\
\hline 10 & Monozygotic twin sister & EDA gene deletion & 270 & 270 & $\mathrm{NI}$ & $\mathrm{NI}$ & - & HED \\
\hline 11 & Proband & c.729_738del7 & 286 & 290 & $74.4 \%$ & $25.6 \%$ & - & HED \\
\hline 15 & Proband & $\mathrm{C} .871 \mathrm{G}>\mathrm{C}$ & 274 & $277(*)$ & $60 \%$ & $40 \%(*)$ & Random & HED \\
\hline 15 & Mother & $\mathrm{C} .871 \mathrm{G}>\mathrm{C}$ & $277(*)$ & 280 & $61.6 \%(*)$ & $38.4 \%$ & Random & Normal \\
\hline 16 & Proband & c.880G $>$ T & 268 & 270 & $43.5 \%$ & $56.5 \%$ & Random & HED \\
\hline 16 & Mother & $(-)$ & 265 & 270 & $19.8 \%$ & $80.2 \%$ & Moderately skewed & Normal \\
\hline 31 & Proband & c.516delC & 271 & 277 & $40 \%$ & $60 \%$ & Random & HED \\
\hline 31 & Mother & $(-)$ & 277 & 277 & $\mathrm{NI}$ & $\mathrm{NI}$ & - & Normal \\
\hline 33 & Proband & Deletion of exon 1 & 277 & 294 & $80.4 \%$ & $19.6 \%$ & Moderately skewed & HED \\
\hline 33 & Mother & $(-)$ & 277 & 283 & $36 \%$ & $64 \%$ & Random & Normal \\
\hline 36 & Proband & c.612delG & 286 & 292 & $76.8 \%$ & $23.3 \%$ & Random & HED \\
\hline 36 & Mother & $(-)$ & 286 & 292 & $55.9 \%$ & $44.1 \%$ & Random & Normal \\
\hline
\end{tabular}

(*) Allele carrier of c.871G > C variant, HED hypohidrotic ectodermal dysplasia, NI non-informative 
Table 3 Allelic variants identified in the EDAR gene

\begin{tabular}{|c|c|c|c|c|c|c|c|c|c|c|c|c|}
\hline Family & Gender & NM & Exon/Intron & Protein & $\begin{array}{l}\text { Affected } \\
\text { domain }\end{array}$ & NM & $\begin{array}{l}\text { Exon/ } \\
\text { Intron }\end{array}$ & Protein & $\begin{array}{l}\text { Affected } \\
\text { domain }\end{array}$ & Origin & $\begin{array}{l}\text { Clinical } \\
\text { Diagnosis }\end{array}$ & $\operatorname{HGMD}(*)$ \\
\hline 5 & $M$ & c. $52-2 A>G$ & Intron 2 & $(-)$ & $\begin{array}{l}\text { Protein } \\
\text { disruption }\end{array}$ & c. $212 \mathrm{G}>\mathrm{A}$ & Exon 4 & p.Cys71Tyr & $\begin{array}{l}\text { TNF } \\
\text { Receptor }\end{array}$ & $\begin{array}{l}\text { Inherited/ } \\
\text { Inherited }\end{array}$ & HED & [3] \\
\hline 18 & M & c. $1072 C>T$ & Exon 9 & p.Arg358* & $\begin{array}{l}\text { Death } \\
\text { domain }\end{array}$ & $(-)$ & $(-)$ & $(-)$ & $(-)$ & Inherited & HED & [26] \\
\hline 42 & M & c. $1073 \mathrm{G}>\mathrm{A}$ & Exon 9 & p.Arg358Gln & $\begin{array}{l}\text { Death } \\
\text { domain }\end{array}$ & $(-)$ & $(-)$ & $(-)$ & $(-)$ & Inherited & HED & [23] \\
\hline 12 & $F$ & c. $1259 \mathrm{G}>\mathrm{A}$ & Exon 12 & p.Arg420GIn & $\begin{array}{l}\text { Death } \\
\text { domain }\end{array}$ & $(-)$ & $(-)$ & $(-)$ & $(-)$ & Inherited & HED & [26] \\
\hline
\end{tabular}

$F$ female, $M$ male, HED hypohidrotic ectodermal dysplasia, (*) Reference in HGMD database

In the $W N T 10 A$ gene, 8 different pathogenic variants were identified in 8 families, three of which were novel variants. A broader phenotypic spectrum was observed in this group of patients, and the most disruptive biallelic WNT10A mutations (in patients with homozygous or compound heterozygous mutations) were associated with Schöpf-Schulz-Passarge syndrome (variants c.18_43del26/ p.Arg7Alafs*28, c.321C > A/p.Cys107* and c.1131C > A/ p.Cys377*). Other significant variants have been found to be associated with HED (compound heterozygous c.27G > A/p.Trp9* and c.92 T > A/p.Leu31Glu) or normohidrotic ED (c.18_43del26/p.Arg7Alafs*28 and c.1131C $>$ A/ p.Cys377*). The recurrent WNTOA variant (c.18_43del26/ p.Arg7Alafs*28) was found in two non-related patients from the same Spanish geographical region. One heterozygous patient was affected by odonto-onycho-dermal dysplasia (OODD) (c.514A > T/p.Arg172Trp) (Table 5).

In the NSTA group, a compound heterozygous patient from family 27 (c.1A > T/p.M1? and c. $321 \mathrm{C}>\mathrm{A} / \mathrm{p}$. Cys $107^{*}$ variants) showed oligodontia and subtle ectodermalrelated symptoms, including thin hair with normal density, periorbital pigmentation and fragile nails. A milder phenotype was observed in two NSTA patients, both of whom had maxillary lateral incisor agenesis, carrying the c.682 T > A/ p.Phe228Ile heterozygous variant, the pathogenicity of which has been established based on the location of the mutation in an important functional domain of the protein; this protein has been implicated in interactions with the membrane receptor and, as consequence, in intra-cellular Wnt signalling [40].

\section{Discussion}

The yield of the analysis of these four genes was $70.8 \%$; specifically, the yield was $76.1 \%$ for HED and $44.4 \%$ for NSTA. Our cohort was similar in size to a French cohort described by Cluzeau et al. [7] with 61 HED patients; however, the four genes accounted for a larger proportion of the French cases (92\%). This discrepancy may be due to recruitment and/or population differences.

$E D A$ variants accounted for most cases (72.5\%), followed by WNT10A (15.7\%), EDARADD (9.8\%) and EDAR (7.8\%). The WNT10A gene should be considered the second candidate gene responsible for ectodermal derivative impairment, in accordance with recently published data for the Italian population [41].

There were more affected males (ratio 5:2) due to a higher prevalence of pathogenic variants in the $E D A$ gene associated with XLHED. The mean age of genetic diagnosis was 5.4 years in children and 40.2 years in adult patients, which was ascertained through the genetic counselling process. The presence of adult patients clinically diagnosed after the age of forty without molecular characterization is significant, reflecting the pre-molecular stage. Taking into account that most detected variants were inherited, we should emphasize the importance of early genetic diagnosis and counselling to prevent new severe cases in affected families and to give these families the opportunity to utilize potential new genetically personalized therapies. Recently, a new prenatal treatment in patients with $E D A$ variants [42] has been described that shows promise for most HED families.

Table 4 Allelic variants identified in the EDARADD gene

\begin{tabular}{|c|c|c|c|c|c|c|c|c|}
\hline Family & Gender & NM & Exon/Intron & Protein & Origin & Clinical Diagnosis & $\operatorname{HGMD}\left({ }^{*}\right)$ & Second variant \\
\hline 21 & M & c. $308 \mathrm{C}>\mathrm{T}$ & Exon 6 & p.Ser103Phe & Inherited & HED & {$[36]$} & EDA: c.572_589del18 \\
\hline 38 & $\mathrm{~F}$ & c. $308 \mathrm{C}>\mathrm{T}$ & Exon 6 & p.Ser103Phe & Inherited & HED & {$[36]$} & \\
\hline 39 & M & c. $308 \mathrm{C}>\mathrm{T}$ & Exon 6 & p.Ser103Phe & Inherited & NSTA & {$[36]$} & WNT10A: c.682 T>A \\
\hline 40 & M & c. $308 \mathrm{C}>\mathrm{T}$ & Exon 6 & p.Ser103Phe & Inherited & HED & {$[36]$} & \\
\hline 51 & M & c. $308 \mathrm{C}>\mathrm{T}$ & Exon 6 & p.Ser103Phe & ND & NSTA & {$[36]$} & EDA: c.866G > A \\
\hline
\end{tabular}

$F$ female, $M$ male, HED, hypohidrotic ectodermal dysplasia, (*) Reference in HGMD database, NSTA non-syndromic tooth agenesis, ND no data 
Table 5 Allelic variants identified in the WNT10A gene

\begin{tabular}{|c|c|c|c|c|c|c|c|c|c|c|}
\hline Family & Gender & NM & Exon/Intron & Protein & NM & Exon/Intron & Protein & Origin & $\begin{array}{l}\text { Clinical } \\
\text { Diagnosis }\end{array}$ & $\operatorname{HGMD}(*)$ \\
\hline 27 & $\mathrm{~F}$ & c. $1 \mathrm{~A}>\mathrm{T}$ & Exon 1 & p.M1? & c. $321 C>A$ & Exon 2 & p.Cys $107^{*}$ & $\begin{array}{l}\text { Inherited/ } \\
\text { Inherited }\end{array}$ & NSTA+ & [37]/ [38] \\
\hline 46 & $\mathrm{~F}$ & $c .27 \mathrm{G}>\mathrm{A}$ & Exon 1 & p.Trp9* & c. $92 \mathrm{~T}>\mathrm{A}$ & Exon 1 & p.Leu31Glu & $\begin{array}{l}\text { Inherited/ } \\
\text { Inherited }\end{array}$ & HED & [38]/Novel \\
\hline 43 & $\mathrm{~F}$ & c.18_43del26 & Exon 1 & p.Arg7Alafs*28 & c.18_43del26 & Exon 1 & p.Arg7Alafs*28 & ND & SSPS & Novel \\
\hline 44 & $\mathrm{~F}$ & c.18_43del26 & Exon 1 & p.Arg7Alafs*28 & c. $1131 C>A$ & Exon 4 & p.Cys $377^{*}$ & $\begin{array}{l}\text { Inherited/ } \\
\text { Inherited }\end{array}$ & $\begin{array}{l}\text { Normohidrotic } \\
\text { ED }\end{array}$ & Novel/Novel \\
\hline 41 & M & C. $514 \mathrm{~A}>\mathrm{T}$ & Exon 2 & p.Arg172Trp & $(-)$ & $(-)$ & $(-)$ & Inherited & OODD & [39] \\
\hline 28 & M & c. $682 \mathrm{~T}>\mathrm{A}$ & Exon 3 & p.Phe228lle & $(-)$ & $(-)$ & $(-)$ & Inherited & NSTA & [38] \\
\hline 39 & M & c. $682 \mathrm{~T}>\mathrm{A}$ & Exon 3 & p.Phe228lle & $(-)$ & $(-)$ & $(-)$ & Inherited & NSTA & [38] \\
\hline 49 & M & c. $321 C>A$ & Exon 2 & p.Cys $107^{*}$ & c. $321 C>A$ & Exon 2 & p.Cys $107^{*}$ & $\begin{array}{l}\text { Inherited/ } \\
\text { Inherited }\end{array}$ & SSPS & [38] \\
\hline
\end{tabular}

$F$ female, $M$ male, HED hypohidrotic ectodermal dysplasia, $\left(^{*}\right)$ Reference in HGMD database, NSTA non-syndromic tooth agenesis, NSTA+ non-syndromic tooth agenesis with other minor ectodermal anomalies, SSPS Schöpf-Schulz-Passarge, ND no data

Our cohort shows a higher allelic heterogeneity of $76.6 \%$ for the four analysed genes, $86.4 \%$ for $E D A$ and $66.6 \%$ for WNT10A; in comparison, the highest published rates are $84 \%$ for $E D A$ [27] and $42.4 \%$ for WNT10A [41].

In regard to the type and location of variants in the $E D A$ gene, four in-frame deletions in the collagen-like domain in exon 4 have been identified (Table 1). Two of them, c.612_629del18 [29] and c.572_589del18 [28], have been previously described to be associated with hypomorphic phenotypes. The other two variants, c.546_581del36 (family 17 ) and c.553_588del36 (family 37 ), also result in less severe signs of HED and nearly normal sweating. In the patients with these variants, the in-frame deletion of the 19 Gly-X-Y repeats in the protein would produce a shorter collagen helix resulting from polymerase slippage but would not affect the multimerization and functionality, causing a milder phenotype [43].

Interestingly, all analysed $E D A$ female carriers showed an HED phenotype that was not linked to skewed Xchromosome inactivation. It is important to note that Xchromosome inactivation was studied in peripheral blood cells instead of skin cells, which may show a different skewed pattern. However, on the other hand, the HED phenotype in these females may suggest that a biological mechanism other than $\mathrm{X}$-inactivation is responsible, in addition to the possible influence of the specifically detected variants and other additional unknown genetic modifiers of clinical expressivity.

Regarding the EDAR variants (Table 3), two of them involve the evolutionally conserved residue Arg358 within the death domain (DD), which has been identified in patients from different continents (c.1072C > T/p.Arg358Ter in American [44] and c.1073G > A/p.Arg358Gln in Asiatic families) [23]. The missense mutation in Arg358 may not affect the interaction with EDARADD [45].
The only identified variant in EDARADD (p.Ser103Phe), which was found in 5 patients, is quite prevalent in the European population. Although the allelic frequency in the healthy population of this variant was $2 \%$ according to the dbSNP database, some authors have suggested that it may make a significant contribution to NSTA but show a low penetrance [29]. In addition, it has been associated with a more severe phenotype in combination with other variants in a heterozygous state in a recent publication [46]. The phenotypes of our patients with p.Ser103Phe variant were consistent with both HED (4/5) and NSTA (1/5) (Table 4). The clinical features in three of them can be explained by the presence of an additional pathogenic variant in EDA and WNT10A. However, in the other two patients with the classical HED phenotype, unknown additional variants in other genes are expected to be involved.

In terms of phenotypes, the $E D A$ gene was most frequently involved in HED (76.6\%) patients, followed by both EDAR and EDARADD and, to a lesser extent, WNT10A. In the Cluzeau cohort [6], the EDA gene accounted for a lower proportion of HED cases (58\%). Our results support the choice to study the EDA gene first, due to its significantly high yield, without using NGS technology in a Spanish patient showing the classical triad of HED symptoms.

The WNT10A gene has been associated with a wide spectrum of ectodermal derivative impairment manifestations, ranging from NSTA to complex rare syndromes such as OODD and SSPS [9, 10, 29, 37, 38, 41, 47-49]. Our findings (Table 5) also reveal that heterozygous variants in WNT10A are associated with NSTA, while homozygous or compound heterozygous variants are linked to a more severe phenotype, either OODD and SSPS, as previously described. Recently, WNT10A- 
linked oligo/hypodontia phenotypes have been reported to be associated with minor ectodermal manifestations, such as mild hair, nails and sweating anomalies [6], as described in our patient from family 27 with tooth agenesis and minor ectodermal signs (NSTA+). For this reason, it is important to take WNT10A into account as a candidate gene for clinical conditions characterized by dental agenesis and other minor ectodermal features, especially in the absence of typical HED facial dysmorphism.

Furthermore, we found that a proportion of cases of tooth agenesis [50], orodental involvement [20] or skin disease [51] may be explained by polygenic inheritance with the co-segregation of multiple variants, which may modulate the final phenotype [52], emphasizing the need to apply more powerful molecular analysis tools during ED diagnosis [39]. In the near future, we will need to understand the pathogenesis and impact of the combination of different allelic variants in different genes in addition to those involved in the Eda or Wnt signalling pathways, mainly by considering that these pathways are associated with specific adaptations in the natural population and that some variants attenuate or increase the final effects; this reflects the relationship between human disease and natural variation, as has been hypothesized [53].

\section{Conclusions}

This is the only molecular study conducted to date in the Spanish ED population, resulting in the specific genetic diagnosis of affected families with HED and NSTA. EDA, EDAR, EDARADD and WNT10A genes constitute the molecular basis of disease in $70.8 \%$ of the patients, with a $74.6 \%$ yield for HED and $44.4 \%$ for NSTA. A high allelic heterogeneity was revealed mainly in EDA, EDAR and WNT10A, for which 12 novel variants were identified. $E D A$ is the most prevalent gene in our cohort, which supports the study of the $E D A$ gene first, due to its significantly high yield, in Spanish patients showing the classical triad of HED symptoms who do not have access to NGS technology. Our data also confirm that the WNT10A gene is the second molecular candidate for involvement in ectodermal derivative impairment, accounting for one-half of non-EDA patients and onethird of NSTA patients.

The broad phenotype spectrum (spanning from classical HED to NSTA) points to the need for a multidisciplinary approach for the care of these patients. The early recognition of these phenotypes and molecular genetic diagnosis in childhood are essential to provide accurate genetic counselling and access to potential new treatments. Further studies using NGS will help to identify the other genes involved in the remaining uncharacterized Spanish patients.

\section{Acknowledgements}

We thank all of the Spanish patients, their families, the Spanish Ectodermal Dysplasia Association (AADE- Asociación de Afectados por Displasia Ectodérmica), and especially Gema Chicano and Ignasi Serrahima for participating in and supporting this study. We extend our gratitude to the following members of GIEDE (Grupo de Investigación Español de Displasias Ectodérmicas), which is a Spanish multidisciplinary research group for ectodermal dysplasia:

- Rebeca Alcalá-García. Servicio de Dermatología. Hospital del Mar, Barcelona. Spain

- Ana Barcia- Ramírez. Hospital Virgen de Valme, Sevilla. Spain

- Jaime Cruz-Rojo. Hospital 12 Octubre. Madrid.Spain

- Blanca Gener-Querol. Servicio Genética. Hospital Universitario de Cruces. Bilbao. Spain.

- Angela Hernández-Martín. Servicio de Dermatología. Hospital Niño Jesús.

Madrid. Spain

- Pablo Lapunzina-Badía, Hospital La Paz. INGEMM, Madrid. Spain

- Isabel Llanos-Rivas. Servicio de Genética. Hospital Universitario de Cruces.

Bilbao. Spain

- Isabel Lorda-Sánchez. Servicio de Genética. Fundación Jiménez Díaz.

Madrid. Spain

- Antonio Martínez-Carrascal. Servicio de Pediatría. Hospital de Requena.

Valencia. Spain

- José-Manuel Mascaró-Galy. Servicio de Dermatología. Hospital Clínico.

Barcelona. Spain

- Lucero Noguera-Morel. Servicio de Dermatología. Hospital Infantil Universitario del Niño Jesús. Madrid. Spain

- María Ángeles Rodríguez-González. Cirugía Máxilofacial. Hospital Clínico

Universitario Virgen de la Arrixaca. Murcia. Spain

- Jaime Sánchez del Pozo. Hospital 12 Octubre. Madrid. Spain

- Verónica Seidel. Genética Pediátrica. Hospital General Universitario Gregorio

Marañón. Madrid. Spain

- Antonio Torrelo. Servicio de Dermatología. Hospital Niño Jesús. Madrid. Spain

- Ma José Trujillo-Tiebas. Servicio de Genética. Fundación Jiménez Díaz. Madrid. Spain

\section{Authors' contributions}

EGN conceived the study; MCMR, MBS and EGN planned the experiments; MJBM, VLG, MJS, LRP, ATSR, MTMM, JFI, PSP and EGN contributed to patient diagnosis and phenotyping; MCMR, PCM and GG performed molecular characterization of patients; EGN, MJBM, VLG, MJS, LRP, ATSR and the GIEDE group contributed to patient recruitment and the acquisition of clinical data; MCMR, MBS and EGN contributed to the analysis of the results; MCMR and EGN wrote the manuscript; all authors provided critical feedback of the analysis and helped to revise the manuscript. All authors read and approved the final manuscript.

\section{Funding}

This project has been supported by the Instituto de Salud Carlos III (ISCIII), Spanish Ministry of Economy and Competitiveness, with grant numbers PI/ 14/01259 and PI/17/00796, and it has been co-funded by FEDER and AADE (Asociación de Afectados por Displasia Ectodérmica).

\section{Availability of data and materials}

The datasets used and/or analysed during the current study are available from the corresponding author upon reasonable request.

\section{Ethics approval and consent to participate}

This study was approved by the Research Ethics Board of the Hospital Clínico Universitario Virgen de la Arrixaca. Informed consent for participation was obtained from all patients.

Consent for publication

Not applicable.

Competing interests

The authors declare that they have no competing interests. 


\section{Author details}

Centro de Bioquímica y Genética Clínica, Hospital Clínico Universitario Virgen de la Arrixaca, IMIB- Arrixaca. Murcia. CIBERER-ISCIII, Madrid, Spain. ${ }^{2}$ Programa de doctorado en Ciencias de la Salud, Universidad Católica de Murcia (UCAM), Murcia, Spain. ${ }^{3}$ Sección Genética Médica. Servicio de Pediatría. Hospital Clínico Universitario Virgen de la Arrixaca. IMIB- Arrixaca, Universidad de Murcia. CIBERER-ISCIII, Madrid, Spain. ${ }^{4}$ Cátedra de Genética. Facultad de Ciencias de la Salud, Universidad Católica de Murcia (UCAM), Murcia, Spain. ${ }^{5}$ Servicio de Dermatología. Hospital Clínico Universitario Virgen de la Arrixaca, Universidad de Murcia, Murcia, Spain. ${ }^{6}$ Departamento de Cirugía, Pediatría, Obstetricia y Ginecología. Facultad de Medicina, Universidad de Murcia, Murcia, Spain. ${ }^{7}$ Sección Genética Médica (Hospital Materno-Infantil. Planta 0), Hospital Clínico Universitario Virgen de la Arrixaca, Ctra. Madrid-Cartagena s/n, El Palmar, CP 30120 Murcia, Spain.

Received: 2 June 2019 Accepted: 5 November 2019

Published online: 03 December 2019

\section{References}

1. Wright JT, Fete M, Schneider H, Zinser M, Koster MI, Clarke AJ, et al. Ectodermal dysplasias: classification and organization by phenotype, genotype and molecular pathway. Am J Med Genet AAm J Med Genet A. 2019;179(3):442-7.

2. Pinheiro $M$, Freire-Maia N. Identifying carriers for X-linked hypohidrotic ectodermal dysplasia. Lancet. 1977;2. England:936.

3. Moya-Quiles MR, Ballesta-Martinez MJ, Lopez-Gonzalez V, Glover G, GuillenNavarro E. A compound heterozygous mutation in the EDAR gene in a Spanish family with autosomal recessive hypohidrotic ectodermal dysplasia. Arch Dermatol ResArch Dermatol Res. 2010;302(4):307-10.

4. Lamartine J. Towards a new classification of ectodermal dysplasias. Clin Exp DermatolClin Exp Dermatol. 2003:28(4):351-5.

5. Visinoni AF, Lisboa-Costa T, Pagnan NA, Chautard-Freire-Maia EA. Ectodermal dysplasias: clinical and molecular review. Am J Med Genet A. 2009;149a(9):1980-2002.

6. Pagnan NA, Visinoni AF. Update on ectodermal dysplasias clinical classification. Am J Med Genet A. 2014;164a(10):2415-23.

7. Cluzeau C, Hadj-Rabia S, Jambou M, Mansour S, Guigue P, Masmoudi S, et al. Only four genes (EDA1, EDAR, EDARADD, and WNT10A) account for $90 \%$ of hypohidrotic/anhidrotic ectodermal dysplasia cases. Hum Mutat. 2011;32(1):70-2.

8. Schmidt-Ullrich R, Paus R. Molecular principles of hair follicle induction and morphogenesis. Bioessays. 2005;27(3):247-61.

9. Castori M, Castiglia D, Brancati F, Foglio M, Heath S, Floriddia G, et al. Two families confirm Schopf-Schulz-Passarge syndrome as a discrete entity within the WNT10A phenotypic spectrum. Clin Genet. 2011;79(1):92-5.

10. Song $S$, Zhao $R, H e ~ H$, Zhang J, Feng $H$, Lin L. WNT10A variants are associated with non-syndromic tooth agenesis in the general population. Hum GenetHum Genet. 2014;133(1):117-24.

11. Song S, Han D, Qu H, Gong Y, Wu H, Zhang X, et al. EDA gene mutations underlie non-syndromic oligodontia. J Dent ResJ Dent Res. 2009;88(2):126-31

12. Gaczkowska A, Abdalla EM, Dowidar KM, Elhady GM, Jagodzinski PP Mostowska A. De novo EDA mutations: variable expression in two Egyptian families. Arch Oral BiolArch Oral Biol. 2016:68:21-8.

13. Koressaar T, Remm M. Enhancements and modifications of primer design program Primer3. Bioinformatics. 2007:23(10):1289-91.

14. Schwarz JM, Cooper DN, Schuelke M, Seelow D. MutationTaster2: mutation prediction for the deep-sequencing age. Nat Methods. 2014;11. United States:361-2.

15. Ng PC, Henikoff S. SIFT: predicting amino acid changes that affect protein function. Nucleic Acids ResNucleic Acids Res. 2003;31(13):3812-4.

16. Adzhubei IA, Schmidt S, Peshkin L, Ramensky VE, Gerasimova A, Bork P, et al. A method and server for predicting damaging missense mutations. Nat Methods. 2010;7. United States:248-9.

17. Choi $Y$, Chan AP. PROVEAN web server: a tool to predict the functional effect of amino acid substitutions and indels. Bioinformatics. 2015;31(16): 2745-7.

18. Richards S, Aziz N, Bale S, Bick D, Das S, Gastier-Foster J, et al. Standards and guidelines for the interpretation of sequence variants: a joint consensus recommendation of the American College of Medical Genetics and
Genomics and the Association for Molecular Pathology. Genet Med. 2015; 17(5):405-24.

19. Jones JR, Nonrandom X. Chromosome inactivation detection. Curr Protoc Hum Genet. 2014:80 Unit 9.7.

20. Prasad MK, Geoffroy V, Vicaire S, Jost B, Dumas M, Le Gras S, et al. A targeted next-generation sequencing assay for the molecular diagnosis of genetic disorders with orodental involvement. J Med Genet. 2016;53. BMA House, Tavistock Square, London, WC1H 9JR:98-110.

21. Stenson PD, Ball EV, Mort M, Phillips AD, Shiel JA, Thomas NS, et al. Human gene mutation database (HGMD): 2003 update. Hum MutatHum Mutat. 2003;21(6):577-81.

22. Vincent MC, Biancalana V, Ginisty D, Mandel JL, Calvas P. Mutational spectrum of the ED1 gene in X-linked hypohidrotic ectodermal dysplasia. Eur J Hum GenetEur J Hum Genet. 2001;9(5):355-63.

23. Shimomura $Y$, Wajid $M$, Weiser J, Kraemer L, Ishii $Y$, Lombillo $V$, et al. Identification of mutations in the EDA and EDAR genes in Pakistani families with hypohidrotic ectodermal dysplasia. Clin Genet. 75. Denmark 2009. p. 582-584.

24. Paakkonen K, Cambiaghi S, Novelli G, Ouzts LV, Penttinen M, Kere J, et al. The mutation spectrum of the EDA gene in X-linked anhidrotic ectodermal dysplasia. Hum MutatHum Mutat. 2001;17(4):349.

25. Schneider P, Street SL, Gaide O, Hertig S, Tardivel A, Tschopp J, et al. Mutations leading to $X$-linked hypohidrotic ectodermal dysplasia affect three major functional domains in the tumor necrosis factor family member ectodysplasin-a. J Biol ChemJ Biol Chem. 2001;276(22):18819-27.

26. Monreal AW, Zonana J, Ferguson B. Identification of a new splice form of the EDA1 gene permits detection of nearly all X-linked hypohidrotic ectodermal dysplasia mutations. Am J Hum GenetAm J Hum Genet. 1998; 63(2):380-9.

27. Lexner MO, Bardow A, Juncker I, Jensen LG, Almer L, Kreiborg S, et al. Xlinked hypohidrotic ectodermal dysplasia. Genetic and dental findings in 67 Danish patients from 19 families. Clin GenetClin Genet. 2008;74(3):252-9.

28. Bayes M, Hartung AJ, Ezer S, Pispa J, Thesleff I, Srivastava AK, et al. The anhidrotic ectodermal dysplasia gene (EDA) undergoes alternative splicing and encodes ectodysplasin-a with deletion mutations in collagenous repeats. Hum Mol GenetHum Mol Genet. 1998;7(11):1661-9.

29. Arte S, Parmanen S, Pirinen S, Alaluusua S, Nieminen P. Candidate gene analysis of tooth agenesis identifies novel mutations in six genes and suggests significant role for WNT and EDA signaling and allele combinations. PLoS OnePLoS One. 2013:8(8):e73705.

30. Li D, Xu R, Huang F, Wang B, Tao Y, Jiang Z, et al. A novel missense mutation in collagenous domain of EDA gene in a Chinese family with $X$ linked hypohidrotic ectodermal dysplasia. J GenetJ Genet. 2015;94(1):115-9.

31. Ruiz-Heiland G, Jabir S, Wende W, Blecher S, Bock N, Ruf S. Novel missense mutation in the EDA gene in a family affected by oligodontia. J Orofac OrthopJ Orofac Orthop. 2016;77(1):31-8.

32. van der Hout AH, Oudesluijs GG, Venema A, Verheij JB, Mol BG, Rump P, et al. Mutation screening of the Ectodysplasin-a receptor gene EDAR in hypohidrotic ectodermal dysplasia. Eur J Hum GenetEur J Hum Genet. 2008; 16(6):673-9.

33. Salas-Alanis JC, Wozniak E, Mein CA, Duran Mckinster CC, Ocampo-Candiani J, Kelsell DP, et al. Mutations in EDA and EDAR genes in a large Mexican Hispanic cohort with Hypohidrotic ectodermal dysplasia. Ann DermatolAnn Dermatol. 2015;27(4):474-7

34. Guazzarotti L, Tadini G, Mancini GE, Giglio S, Willoughby CE, Callea M, et al. Phenotypic heterogeneity and mutational spectrum in a cohort of 45 Italian males subjects with X-linked ectodermal dysplasia. Clin Genet. 2015;87(4):338-42.

35. Cluzeau C, Hadj-Rabia S, Bal E, Clauss F, Munnich A, Bodemer C, et al. The EDAR370A allele attenuates the severity of hypohidrotic ectodermal dysplasia caused by EDA gene mutation. Br J Dermatol. 2012;166(3):678-81.

36. Bergendal B, Klar J, Stecksen-Blicks C, Norderyd J, Dahl N. Isolated oligodontia associated with mutations in EDARADD, AXIN2, MSX1, and PAX9 genes. Am J Med Genet A. 2011;155a(7):1616-22.

37. Plaisancie J, Bailleul-Forestier I, Gaston V, Vaysse F, Lacombe D, HolderEspinasse $M$, et al. Mutations in WNT10A are frequently involved in oligodontia associated with minor signs of ectodermal dysplasia. Am J Med Genet A. 2013;161a(4):671-8

38. Bohring A, Stamm T, Spaich C, Haase C, Spree K, Hehr U, et al. WNT10A mutations are a frequent cause of a broad spectrum of ectodermal dysplasias with sex-biased manifestation pattern in heterozygotes. Am J Hum GenetAm J Hum Genet. 2009;85(1):97-105. 
39. Retterer K, Juusola J, Cho MT, Vitazka P, Millan F, Gibellini F, et al. Clinical application of whole-exome sequencing across clinical indications. Genet Med. 2016;18(7):696-704.

40. Trzeciak WH, Koczorowski R. Molecular basis of hypohidrotic ectodermal dysplasia: an update. J Appl Genet. 2016;57(1):51-61.

41. Guazzarotti L, Tadini G, Mancini GE, Sani I, Pisanelli S, Galderisi F, et al. WNT10A gene is the second molecular candidate in a cohort of young Italian subjects with ectodermal derivative impairment (EDI). Clin Genet. 2018;93(3):693-8.

42. Schneider H, Faschingbauer F, Schuepbach-Mallepell S, Korber I, Wohlfart S, Dick A, et al. Prenatal correction of X-linked Hypohidrotic ectodermal dysplasia. N Engl J MedN Engl J Med. 2018;378(17):1604-10.

43. Dietz J, Kaercher T, Schneider AT, Zimmermann T, Huttner K, Johnson R, et al. Early respiratory and ocular involvement in $\mathrm{X}$-linked hypohidrotic ectodermal dysplasia. Eur J PediatrEur J Pediatr. 2013;172(8):1023-31.

44. Jorgenson RJ, Dowben JS, Dowben SL. Autosomal dominant ectodermal dysplasia. J Craniofac Genet Dev Biol. 1987;7(4):403-12.

45. Masui Y, Farooq M, Sato N, Fujimoto A, Fujikawa H, Ito M, et al. A missense mutation in the death domain of EDAR abolishes the interaction with EDARADD and underlies hypohidrotic ectodermal dysplasia. Dermatology. 2011;223(1):74-9.

46. Barbato E, Traversa A, Guarnieri R, Giovannetti A, Genovesi ML, Magliozzi $M R$, et al. Whole exome sequencing in an Italian family with isolated maxillary canine agenesis and canine eruption anomalies. Arch Oral BiolArch Oral Biol. 2018;91:96-102.

47. Tardieu C, Jung S, Niederreither K, Prasad M, Hadj-Rabia S, Philip N, et al. Dental and extra-oral clinical features in 41 patients with WNT10A gene mutations: a multicentric genotype-phenotype study. Clin Genet. 2017;92(5):477-86.

48. Bergendal B, Norderyd J, Zhou X, Klar J, Dahl N. Abnormal primary and permanent dentitions with ectodermal symptoms predict WNT10A deficiency. BMC Med Genet. 2016;17(1):88.

49. Arzoo PS, Klar J, Bergendal B, Norderyd J, Dahl N. WNT10A mutations account for (1/4) of population-based isolated oligodontia and show phenotypic correlations. Am J Med Genet A. 2014;164a(2):353-9.

50. Dinckan N, Du R, Petty LE, Coban-Akdemir Z, Jhangiani SN, Paine I, et al. Whole-exome sequencing identifies novel variants for tooth agenesis. J Dent ResJ Dent Res. 2018:97(1):49-59.

51. Takeichi T, Nanda A, Liu L, Salam A, Campbell P, Fong K, et al. Impact of next generation sequencing on diagnostics in a genetic skin disease clinic. Exp Dermatol. 2013;22(12):825-31.

52. Wohlfart S, Hammersen J, Schneider H. Mutational spectrum in 101 patients with hypohidrotic ectodermal dysplasia and breakpoint mapping in independent cases of rare genomic rearrangements. J Hum GenetJ Hum Genet. 2016;61(10):891-7.

53. Sadier A, Viriot L, Pantalacci S, Laudet V. The ectodysplasin pathway: from diseases to adaptations. Trends GenetTrends Genet. 2014;30(1):24-31.

\section{Publisher's Note}

Springer Nature remains neutral with regard to jurisdictional claims in published maps and institutional affiliations.

Ready to submit your research? Choose BMC and benefit from:

- fast, convenient online submission

- thorough peer review by experienced researchers in your field

- rapid publication on acceptance

- support for research data, including large and complex data types

- gold Open Access which fosters wider collaboration and increased citations

- maximum visibility for your research: over $100 \mathrm{M}$ website views per year

At $\mathrm{BMC}$, research is always in progress.

Learn more biomedcentral.com/submissions 